\title{
Aerodynamics of Heat Exchangers for High-Altitude Aircraft Mark Drela
}

Reprinted from

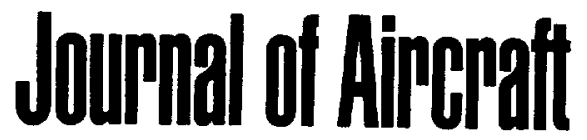

Volume 33, Number 1, Pages 176-184

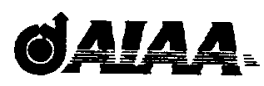

American Institute of Aeronautics and Astronautics, Inc. 


\title{
Aerodynamics of Heat Exchangers for High-Altitude Aircraft
}

\author{
Mark Drela* \\ Massachusetts Institute of Technology, Cambridge, Massachusetts 02139
}

\begin{abstract}
Reduction of convective heat transfer with altitude dictates unusually large heat exchangers for pistonengined high-altitude afreraft. The relatively large alreraft drag fraction ssociated wh cooling at high altitudes makes the efficient desigo of the entire heat exchanger installation an essentiol part of the aircraft's aerodynamic design. The parameters that directly influence cooling drag are developed in the context of high-altitude flight. Candidate wing airfolls that incorporate hent exchangers are examined. Such integrated wing-airfoil/heat-exchanger installations appear to be attractive alternatives to isolated heat-exchanger installations. Examples are drawn from integrated installations on existing or planned high-altitude aircraft.
\end{abstract}

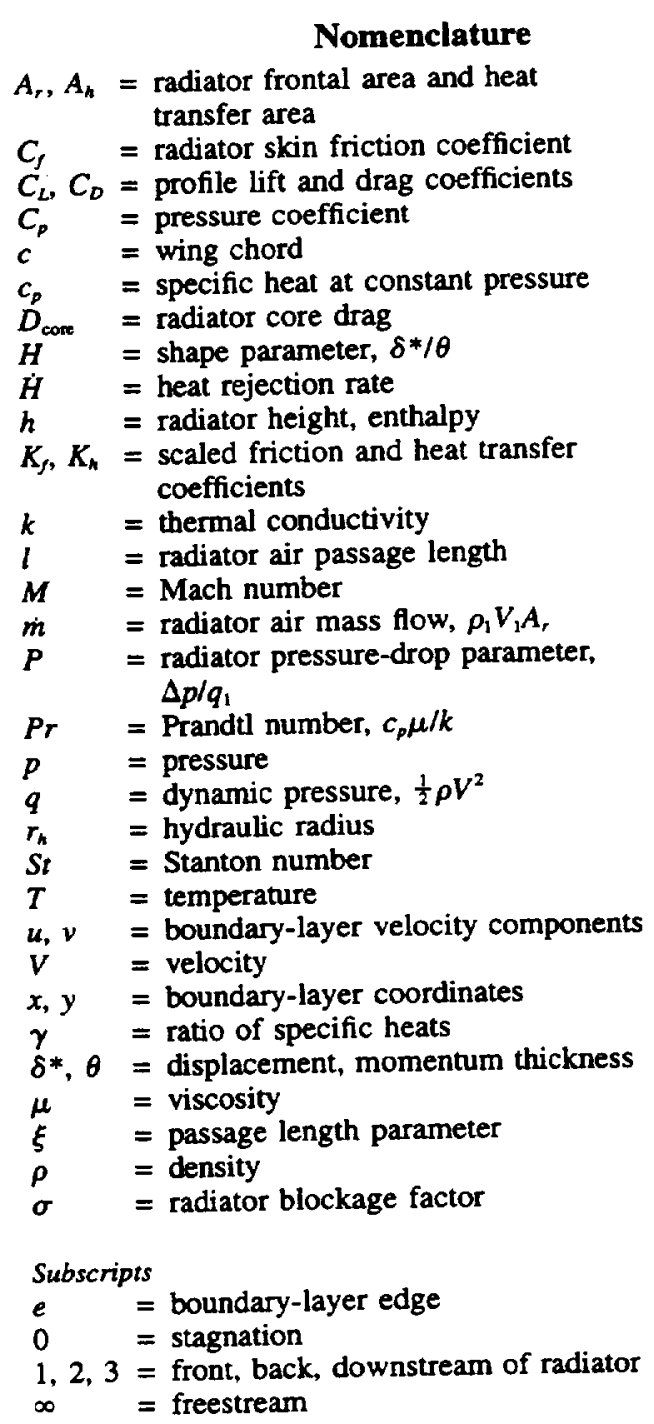

Received May 8, 1995; presented as Paper 95-1866 at the AIAA 13th Applied Aerodynamics Conference, San Diego, CA, June 1922, 1995; revision received Aug. 19, 1995; accepted for publication Aug. 21,1995 . Copyright $\odot 1995$ by the American Institute of Aeronautics and Astronautics, Inc. All rights reserved.

*Associate Professor, Department of Aeronautics and Astronautics, Room 37-475. Associate Fellow AIAA.

\section{Introduction}

Q UBSONIC ultrahigh-altitude aircraft are currently being developed for use in a variety of missions, notably for in situ atmospheric science measurements. ${ }^{1-3}$ Although previous high-altitude aircraft have not flown above $70,000 \mathrm{ft}$ (e.g., Boeing's Condor $\left.{ }^{4}\right)$, cruise altitudes of up to $30 \mathrm{~km}(100,000$ $\mathrm{ft}$ ) are being considered for the new science mission-oriented aircraft. Practical constraints of low development and unit cost, and the fact that turbofan and turboprop engines begin to suffer large power losses near $20 \mathrm{~km}(65,000 \mathrm{ft})$, dictate the use of turbocharged reciprocating engines for such aircraft. A reciprocating engine used at high altitudes must in practice be liquid cooled and the size of the radiator must be considerably larger at high altitude than at sea level. Russ et al. ${ }^{3}$ have outlined the system integration issues involved in the development of such radiators. Careful attention must be paid to the aerodynamic design of the radiator installation, particularly for long-range aircraft whose performance is sensitive to the associated parasite drag.

The large radiators typically required for high-altitude aircraft can adversely impact the flow around neighboring aerodynamic components. For example, in the case of a wing leading-edge inlet, an ineffective inlet geometry can have very adverse effects on the wing airfoil, as demonstrated in previous experimental studies. ${ }^{67}$ This makes an isolated nacelle radiator installation less risky and more attractive. On the other hand, effective integration of the radiator with the wing airfoil also offers possibilities for favorable interactions and greater compactness, with possibly lower overall drag and lower system weight than with an equivalent isolated installation.

This article examines the aerodynamics of closely coupled aifoil/radiator installations and develops criteria for selecting important installation and operating parameters. A computational two-dimensional method for predicting performance of the entire airfoil/radiator configuration will be used to illustrate the pros and cons of several alternative configurations being employed on operational and planned ultrahigh-altitude aircraft.

\section{Characteristics of Low-Drag Heat Exchanger Installations}

The ideas behind low drag radiator installations have been known since before World War II (see Ref. 8). Hoerner' discusses installations employed to date. The basic principle is to decelerate the cooling airflow, pass it through the radiator at low speed, and then accelerate it back to ambient pressure as shown in Fig. 1. In practice, the final streamtube area $A_{3}$ is very close to the geometric nozzle area. 

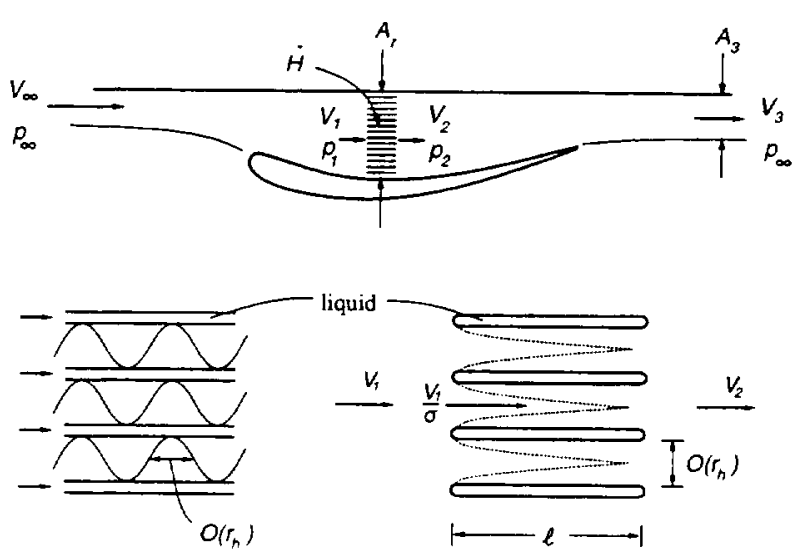

Fig. 1 Cowled radiator and radiator core section.

The drag $D_{\text {core }}$ of the radiator core is defined as the cooling airflow's rate of change of momentum:

$$
D_{\text {core }}=\dot{m}\left(V_{\infty}-V_{3}\right)=\rho_{1} V_{1} A_{r}\left(V_{\infty}-V_{3}\right)
$$

Adding to this will be the profile drag of the cowl $D_{\text {cowl }}$, and perhaps some interference drag due to unfavorable interactions. In a well-designed heat exchanger system, the $D_{\text {core }}$ and $D_{\text {cowl }}$ drag components should be predominant, and will be the primary topic here.

\section{A. Radiator Core Geometry}

In most liquid/air radiator cores, such as those found in automobiles, the liquid coolant flows perpendicular to the airflow in flattened tubes that are separated by corrugated metal fins that form cooling-air passages, as shown in Fig. 1. The crosssectional size of the air passages is commonly characterized by $r_{h}$, defined in terms of the frontal/heat transfer area ratio, and the streamwise flow length $l$ (i.e., the thickness) of the radiator:

$$
r_{h} \equiv l \frac{\sigma A_{r}}{A_{h}}=\frac{\text { passage area }}{\text { passage perimeter }}
$$

This $r_{h}$ definition reduces to half the passage width for a long rectangular section and to one-quarter of the passage diameter for a circular cross section. The presence of the liquid-carrying tubes, represented by $\sigma$, results in the flow in the passage inlet being increased from $V_{1}$ to $V_{1} / \sigma$. For most liquid/air radiators, $\sigma$ is close to unity.

For a compact radiator it is desirable to increase the internal radiator wetted area $A_{h}$ by decreasing $r_{h}$ and/or increasing $l$. However, once the thermal and momentum boundary layers begin to merge, as shown in Fig. 1, further increases in heat transfer are smaller compared to the increases in skin friction and radiator pressure drop. This effect can be examined via the average friction coefficient and Stanton number:

$$
\begin{gathered}
C_{f}=\frac{2 \bar{\tau}}{\rho_{1}\left(V_{1} / \sigma\right)^{2}}=\frac{\sigma A_{r}}{A_{h}} \frac{2 \Delta \bar{p}}{\rho_{1}\left(V_{1} / \sigma\right)^{2}} \\
S t=\frac{\dot{H} / A_{h}}{C_{p}\left(T_{r}-T_{1}\right) \rho_{1} V_{1} / \sigma}
\end{gathered}
$$

The average shear stress $\bar{\tau}$ is related to the pressure drop corrected for the increasing momentum flux of the developing velocity profile in the passage

$$
\Delta \bar{p}=\Delta\left(p+\int \rho V^{2} \frac{\mathrm{d} A}{A}\right)
$$

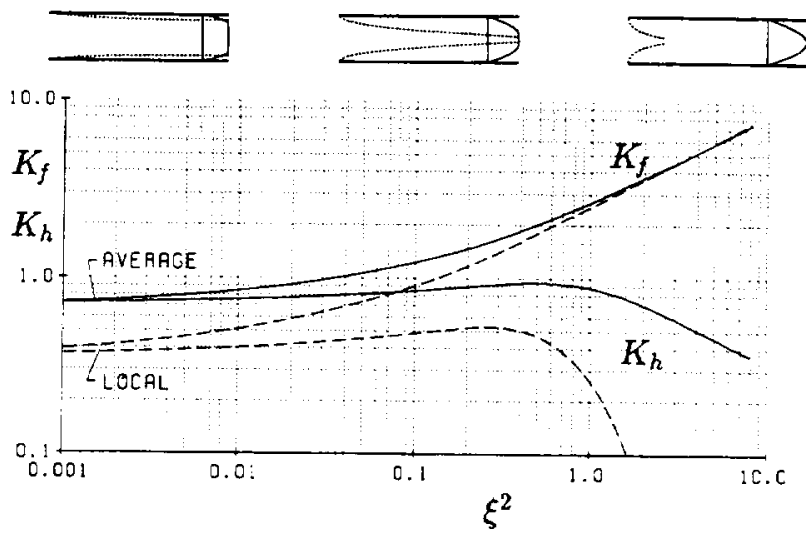

Fig. 2 Average and local Reynolds number-scaled heat transfer and skin friction coefficients vs scaled passage length parameter for a two-dimensional channel.

So that for the limiting case of zero blockage $(\sigma=1)$, the net pressure drop across the radiator would be $\Delta \bar{p}$.

Figure 2 shows local and average Reynolds number-scaled friction and heat transfer coefficients

$$
\begin{gathered}
K_{f}=\frac{C_{f}}{2}\left(\frac{\rho_{1} V_{1} l}{\sigma \mu_{1}}\right)^{1 / 2} \\
K_{h}=\operatorname{StPr}^{2 / 3}\left(\frac{\rho_{1} V_{1} l}{\sigma \mu_{1}}\right)^{1 / 2}
\end{gathered}
$$

vs the scaled $\xi$

$$
\xi^{2} \equiv \frac{l}{r_{h}} \frac{\sigma}{\operatorname{Pr}_{r}} \frac{\mu_{1}}{\rho_{1} V_{1} r_{h}}
$$

for laminar entrance flow in a two-dimensional channel. The curves were computed using a finite difference thin shear layer code with a small Mach number and $\operatorname{Pr}=0.72$, although the dependence on the Prandtl number is very weak. The limiting case $\xi \rightarrow 0$ corresponds to laminar fiat plate flow, for which

$$
K_{f}=K_{h}= \begin{cases}0.664 & \text { average } \\ 0.332 & \text { local }\end{cases}
$$

with $K_{f}=K_{h}$ constituting the Reynolds analogy.

Past $\xi=1.0$, which is comparable to the entrance-flow length for a two-dimensional channel, the friction begins to increase more rapidly, while the heat rejection begins to level off and eventually decrease. This then sets a practical upper limit for the length of the radiator and often is the limit of measured $C_{f}$ and $S t$ radiator core data, such as that presented in Kays and London. ${ }^{10}$ Core data for real radiator geometries differ quantitatively somewhat from the curves in Fig. 2, but still have the same general behavior.

The choice of $\xi \simeq O(1)$, which defines an entrance-flow radiator, is likely to be effective in a high-altitude aircraft where it is necessary to minimize the inevitably large radiator frontal area, but without incurring an excessive pressure drop. In any situation, reducing the radiator drag can be achieved with a smaller $\xi$, while obtaining a more compact radiator with a smaller frontal area can be achieved with a larger $\xi$.

\section{B. Pressure Loss - Drag Relation}

For the case of low freestream Mach number and frictionless radiator, Rauscher" has explicitly related $V_{3}$ and the drag $D_{\text {core }}$ to the heat rejection and exit area. For fully compressible flow, the dependence of the radiator drag on all of the operating parameters cannot be explicitly written down, but is implicitly determined by the governing equations for compressible quasi- 
one-dimensional channel flow with friction and heat addition, as discussed in McGeer et al. ${ }^{12}$ and Russ et al. ${ }^{5}$ These equations can be readily solved by Newton iteration for the flow variables $(\rho, p, V)_{1.2 .3}$. The numerical two-dimensional simulations presented later duplicate such fully compressible results. Here, Rauscher's low-speed treatment will be extended to nonzero friction, to clearly identify the relevant parameters that influence the radiator drag.

Rauscher assumes that the only significant density change occurs across the radiator,

$$
\rho_{1}=\rho_{\text {oo, }}, \quad \rho_{2} \approx \rho_{3}
$$

and employs the incompressible Bernoulli equation upstream and downstream of the radiator. His momentum equation across the radiator is extended here to include the frictional pressure drop parameter $P$, which is experimentally measured for a given radiator core and Reynolds number. For numerical simulation purposes, $P$ is separated into the core inlet $P_{b}$ core exit $P_{e}$, friction $P_{f}$, and acceleration terms, as suggested by the pressure-drop model of Kays and London ${ }^{30}$ :

$$
\begin{gathered}
p_{1}-p_{2}=\frac{1}{2} \rho_{1} V_{1}^{2} P \\
P=P_{i}+\frac{V_{2}}{V_{1}} P_{e}+\left(1+\frac{\mu_{2} V_{2}}{\mu_{1} V_{1}}\right) \frac{P_{f}}{2}+2\left(\frac{V_{2}}{V_{1}}-1\right)
\end{gathered}
$$

The friction term is often dominant and is simply related to the average skin friction coefficient and blockage factor:

$$
P_{f}=\left(C_{f} / \sigma^{2}\right)\left(U / r_{h}\right)
$$

Typical overall pressure drop parameter values for liquid/air radiator cores are in the range $5<P<20$.

In his frictionless model, Rauscher employed only the acceleration term

$$
P=2\left[\left(V_{2} / V_{1}\right)-1\right]
$$

and gave explicit (and elaborate) expressions for $V_{1}, V_{3}$, and $D_{\text {core }}$ for a given radiator, exit area, flight conditions, and heat rejection. For design purposes, it is more useful to consider the radiator velocity ratio $V_{1} / V_{\infty}$ as a free parameter, since this controls the radiator air mass flow, and immediately gives the radiator velocity and density ratios:

$$
\frac{V_{2}}{V_{1}}=\frac{\rho_{1}}{\rho_{2}}=\frac{T_{2}}{T_{1}} \approx 1+\frac{\dot{H}}{\dot{m}} \frac{\gamma-1}{\gamma} \frac{\rho_{1}}{p_{1}}
$$

The net pressure drop parameter $P$ can then be estimated from the Kays and London model [Eq. (9)], or preferably taken from measured data if available. Combining the radiator momentum Eq. (8) with the inlet and outlet Bernoulli and continuity equations finally gives the exit velocity

$$
\left(V_{3} / V_{\infty}\right)^{2}=\left(\rho_{1} / \rho_{2}\right)\left[1-P\left(V_{1} / V_{\infty}\right)^{2}\right]
$$

and the radiator drag then follows from its definition [Eq. (1)].

A useful further approximation is to consider the case of a small exit velocity defect and small density change,

$$
V_{3} / V_{\infty} \approx 1, \quad \rho_{1} / \rho_{2} \approx 1
$$

in which case we have

$$
\frac{V_{3}}{V_{\infty}} \approx 1-\frac{1}{2} P\left(\frac{V_{1}}{V_{\infty}}\right)^{2}+\frac{\gamma-1}{2} \frac{\dot{H}}{\dot{m}} \frac{\rho_{1}}{\gamma p_{1}}
$$

and the drag power and equivalent radiator drag coefficient can then be estimated as

$$
\begin{gathered}
D_{\text {core }} V_{\infty} \simeq \frac{\dot{m}}{2} V_{\infty}^{2} P\left(\frac{V_{1}}{V_{\infty}}\right)^{2}-\dot{H} \frac{\gamma-1}{2} M_{\infty}^{2} \\
C_{D_{\infty}}=\frac{2 D_{\infty \infty r r}}{\rho_{\infty} V_{\infty}^{2} S} \simeq \frac{A_{r}}{S}\left[P\left(\frac{V_{1}}{V_{\infty}}\right)^{3}-\frac{\dot{H}}{\rho_{\infty} V_{\infty}^{3} A_{r}}(\gamma-1) M_{\infty}^{2}\right]
\end{gathered}
$$

where the approximation $\rho_{1} / p_{1}=\rho_{\infty} / p_{\infty}$ has been made. Equations (14) and (15) clearly identify the pressure coefficient drop across the radiator

$$
\Delta C_{p_{1}}=C_{p_{1}}-C_{p_{2}}=P\left(V_{1} / V_{\infty}\right)^{2}
$$

as the decisive parameter in controlling radiator drag, since the mass flow $\dot{m} \sim A_{r} V_{1}$ is strongly constrained by the requirement to reject the required heat load. The second term, proportional to $H M_{\infty}^{2}$, is the ramjet thrust, which may significantly offset the first friction drag term in some installations.

\section{Heat Transfer-Drag Relation}

Sizing of the radiator requires a quantitative relation between the known heat load $\dot{H}$ and the other radiator parameters, and the mass flow $m=\rho_{1} V_{1} A_{r}$ in particular. The experimental data in Kays and London ${ }^{10}$ indicate that for typical plate/fin radiators in the laminar flow regime, the skin friction and heat transfer coefficients closely follow the Reynolds number-dependent scaling implied in Fig. 2. Combining Eqs. (2) and (4) gives a direct relation between heat rejection and mass flow:

$$
\dot{H}=\xi K_{h} c_{p}\left(T_{r}-T_{1}\right) P r^{-1 / 6} \rho_{1} V_{1} A_{r}
$$

From Fig. 2, the scaled Stanton number takes on the values $K_{h} \simeq 0.7-0.9$ for a two-dimensional passage, but in practice would be taken from measured data for a particular radiator core.

The fully developed flow case $\xi \gg 1$, considered by Capon, has a clear loss in effectiveness (smaller $\boldsymbol{K}_{h}$ ) due to the passage centerline temperature asymptoting to the radiator metal temperature $T_{r}$, at which point further heat transfer cannot occur. The $T_{r}$ itself can vary in the range between the incoming air temperature and the liquid coolant temperature

$$
T_{1}<T_{r}<T_{\text {uquad }}
$$

depending on the coolant circulation rate. Of course, $T_{r}$ can also vary across the radiator if the liquid coolant is not circulated and mixed rapidly. These effects cannot be considered here, but can in general be lumped into a net effective $K_{h}$.

The entrance-flow radiator heat rejection relation [Eq. (17)], combined with the radiator drag definition [Eq. (1)], results in a direct relation between the heat rejection and drag power:

$$
D_{\text {cose }} V_{\infty}=\frac{\dot{H} V_{\infty}^{2} P r^{1 / 6}}{\xi K_{n} c_{p}\left(T_{r}-T_{1}\right)}\left(1-\frac{V_{3}}{V_{\infty}}\right)
$$

Using the small-defect approximation [Eq. (12)], this becomes

$$
D_{\text {core }} V_{\infty} \simeq \dot{H}\left[\frac{V_{\infty}^{2} P^{1 / 6}}{\xi K_{h} c_{p}\left(T_{r}-T_{1}\right)} \frac{P}{2}\left(\frac{V_{1}}{V_{\infty}}\right)^{2}-\frac{\gamma-1}{2} M_{\infty}^{2}\right]
$$

If the friction term in Eq. (9) is the dominant contribution to $P$, as is usually the case, the drag power can be further simplified to

$$
D_{\text {core }} V_{\infty} \simeq \dot{H}\left[\frac{V_{\infty}^{2} P r^{2 / 3}}{c_{p}\left(T_{r}-T_{1}\right)} \frac{1}{\sigma^{2}} \frac{K_{f}}{K_{h}}\left(\frac{V_{1}}{V_{\infty}}\right)^{2}-\frac{\gamma-1}{2} M_{\infty}^{2}\right]
$$



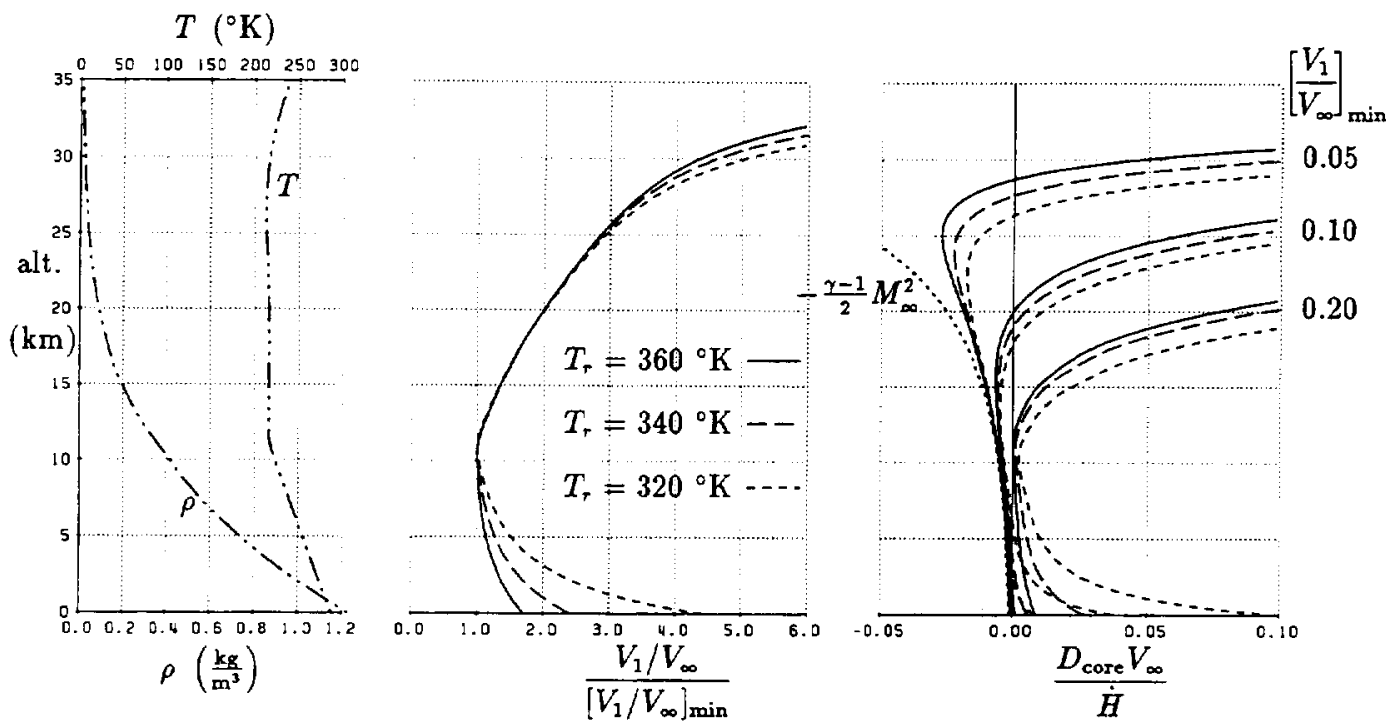

Fig. 3 U.S. Standard Atmosphere density and temperature profiles, corresponding radiator velocity ratio required for cooling regulation, and associated drag power vs altitude for three radiator temperatures and three minimum velocity ratios. Assumes $\frac{1}{2} \rho_{-} V_{-}^{2}=480 \mathrm{~Pa}^{2}=$ $10 \mathrm{lb} / \mathrm{ft}^{2}$ and $K_{f} / \sigma^{2} K_{k}=10$.

Equation (21) quantifies the benefits of using a small velocity ratio $V_{1} / V_{\infty}$ of selecting a radiator core with a small $K_{f} / K_{h}$ ratio and near-unity blockage factor $\sigma$, and of using a large radiator temperature $T_{r}$.

\section{Cooling Regulation Considerations}

The discussion up to now has been aimed at the sizing of an ideal radiator for a particular altitude. In practice, the radiator is of one fixed size, but it must still reject heat at the same rate at all altitudes, such as in a typical full-power climbout. Recasting Eq. (17) as

$$
\dot{H}=K_{h} \operatorname{Pr}^{-2 / 3} \frac{l}{r_{h}} c_{p}\left(T_{r}-T_{1}\right)\left(\frac{\sigma \mu_{1}}{\rho_{1} V_{1} l}\right)^{1 / 2} \rho_{1} V_{1} A_{r}
$$

shows that for a given radiator geometry and heat load, it is necessary to hold

$$
\left(T_{r}-T_{1}\right)\left(\rho_{1} V_{1} \mu_{1}\right)^{1 / 2} \sim \text { const }
$$

with altitude and dynamic pressure.

Constraint (23) can be satisfied either by regulating $T$, (by varying the liquid coolant circulation rate), or by regulating the radiator velocity ratio $V_{1} / V_{\infty}$ (by varying the nozzle area $A_{3}$ with a cowl flap). Regulating the coolant flow is undesirable in practice, since at low circulation rates the engine may encounter large thermal stresses from the returning excessively cold coolant, or the coolant may freeze and block the radiator. In contrast, regulating the velocity ratio permits maintaining the same ideal coolant temperature with varying altitude and is much more attractive for reliable engine operation.

It is reasonable to assume that the conditions at station 1 are nearly at stagnation:

$$
\rho_{1} \simeq \rho_{0}=\rho_{\infty}\left\{1+[(\gamma-1) / 2] M_{\infty}^{2}\right\}^{L /(\gamma-1)}
$$

Hence, constraint (23) implies that for proper cooling regulation it is necessary to vary the radiator velocity ratio as

$$
\frac{V_{1}}{V_{\infty}} \sim \frac{\left\{1+[(\gamma-1) / \gamma]\left(q_{\infty} / p_{\infty}\right)\right\}^{-\{\gamma /(\gamma-1)}}{\left(T_{r}-T_{\infty}\left\{1+[(\gamma-1) / \gamma]\left(q_{\infty} / p_{\infty}\right)\right\}\right)^{2}\left(\rho_{\infty} q_{\infty}\right)^{1 / 2} \mu_{\infty}}
$$

using $\gamma M^{2}=2 q / p$ and assuming that $\mu \sim T$. A more accurate $\mu(T)$ relation could be used. Figure 3 shows the required ve- locity ratio defined by $\mathrm{Eq}$. (24) relative to its minimum value as a function of altitude, for three radiator temperatures and at fixed dynamic pressure. The radiator temperature does not significantly impact the range of $V_{1} / V_{\infty}$ required for cooling regulation, but it affects the heat rejection per frontal area. In all cases, flight near the tropopause has the largest available cooling and will likely be the design point for the smallest $V_{1} / V_{\infty}$ tolerable by the heat exchanger installation.

To determine the radiator drag variation with altitude, it is necessary to require that the velocity ratio $V_{1} / V_{\infty}$ in $\mathrm{Eq}$. (20) varies with altitude according to the regulation constraint (24). The approximate drag-power Eq. (21) can be expressed as

$$
\begin{aligned}
& \frac{D_{\text {cose }} V_{\infty}}{\dot{H}}=\frac{\left(2 q_{\infty} / p_{\infty}\right) P r^{2 / 3}}{c_{p}\left(T_{r}-T_{\infty}\left\{1+[(\gamma-1) / 2] M_{\infty}^{2}\right\}\right)} \frac{1}{\sigma^{2}} \frac{K_{f}}{K_{h}}\left(\frac{V_{1}}{V_{\infty}}\right)^{2} \\
& -\frac{\gamma-1}{\gamma} \frac{q_{\infty}}{p_{\infty}}
\end{aligned}
$$

with $V_{1} / V_{\infty}$ determined by Eq. (24) once the minimum value $\left(V_{1} / V_{\infty}\right)_{\min }$ is chosen. Since $H$ is typically comparable to mechanical engine power, Eq. (25) gives the fractional power loss (or gain) due to radiator drag or thrust. This is plotted in Fig. 3 for the three radiator temperatures and three minimum velocity ratios. The negative ramjet term in Eq. (25) is also shown. The curves were generated assuming

$$
\frac{1}{\xi K_{h}} \frac{P}{2} \approx \frac{1}{\sigma^{2}} \frac{K_{f}}{K_{h}}=10
$$

which is typical for liquid/air heat exchangers. Rather small minimum velocity ratios at the tropopause are required if the radiator drag power is to be kept at a reasonably small level at much higher altitudes. Note also that for such designs, a net thrust is obtained from the ramjet term $-[(\gamma-1) / 2] M_{\text {o over }}^{2}$ much of the operating altitudes. The major drawback to small minimum velocity ratios $V_{1} / V_{\infty}$ is that $A_{r}$ scales more or less inversely with $V_{1}$ for a given heat rejection rate, as can be seen from Eq. (17). This implies proportionately larger cooling system weight and larger duct cowls that also extract a proportionately larger profile drag penalty. Clearly, a careful tradeoff between these design factors is required. 


\section{Numerical Simulation}

The numerical simulations presented here were generated with an extension of the MSES viscous/inviscid two-dimensional multielement airfoil code. ${ }^{13}$ It employs a finite volume Euler formulation discretized on an intrinsic streamline grid. The viscous layers are represented by a two-equation laggeddissipation integral formulation. The two formulations are strongly coupled through the displacement thickness and solved simultaneously by a global Newton method.

\section{A. Inviscid Flow Model}

The radiator in the simulation is placed along a row of cells that span the gap between two airfoil elements. Equation (8) together with the $P$ definition [Eq. (9)] replaces the streamwise momentum equation at each radiator cell. The normal-momentum equation is replaced by the requirement that the flow direction at the exit (station 2) is along a specified direction, typically normal to the radiator face. This models the flowstraightening effect of the radiator core passages.

The heat addition is simulated by specifying the stagnation enthalpy all along the streamtubes behind the radiator to be increased by an amount $\Delta h_{0}$.

$$
h_{0_{2}}-h_{0_{1}} \equiv \Delta h_{0}=\dot{H} / \dot{m}
$$

The heat rejection rate $H$ is prescribed, while the radiator mass flow $m$ is, in general, unknown, and is generated as part of the solution. The temperature jump

$$
\Delta T=\left\{\Delta h_{0}+\left[\left(V_{1}^{2}-V_{2}^{2}\right) / 2\right]\right\}\left(1 / c_{p}\right)
$$

is therefore a result of the solution as well. If it is found that the downstream temperature exceeds the coolant temperature, i.e.,

$$
T_{2}=T_{1}+\Delta T>T_{\text {liquid }}
$$

then an unrealistically large $\dot{H}$ is being specified.

\section{B. Viscous Flow Model}

A very important effect that must be modeled in a numerical simulation is the radiator's thinning effect on the surface boundary layers that pass through it. Mehta ${ }^{14}$ describes this effect for screens used to suppress separation in diffusers. Figure 4 shows the pressure field set up by the radiator and the incoming boundary layer. Near the wall where the velocity is small, little or no pressure drop can be supported across the radiator, resulting in the low downstream pressure $p_{2}$ being felt in front of the radiator and accelerating the upstream boundary layer. The usual thin shear-layer approximation $\partial p / \partial y=0$ is obviously strongly violated, since at the upstream radiator face $\left(p_{e}-p_{\text {wat }}\right) / \rho_{e} \mu_{e}^{2}=P / 2$, which is typically much greater than unity. However, the effect can be estimated by adding an assumed radiator-induced pressure gradient term to the usual thin shear-layer momentum equation:

$$
\rho u \frac{\partial u}{\partial x}+\rho v \frac{\partial u}{\partial y}=-\frac{d p_{c}}{d x}+\frac{\partial \tau}{\partial y}+\left(1-\frac{\rho u^{2}}{\rho_{e} u_{2}^{2}}\right) \Delta p \frac{d}{d x} e^{(x-x) y \delta}
$$

The radiator-induced pressure field sketched in Fig. 4 is assumed to propagate upstream with the length scale $\delta$ comparable to the boundary-layer thickness and to scale with the local dynamic pressure $\rho u^{2}$. Since the resulting acceleration of the boundary layer is an inviscid mechanism, the standard approximation of neglecting streamwise diffusion is retained.

Equation (28) produces the following modified integral momentum and kinetic energy shape parameter equations:

$$
\frac{\mathrm{d}}{\mathrm{d} x} \ln \left(\rho_{\mathrm{e}} u_{\mathrm{e}}^{2} \theta\right)=\frac{c_{f}}{2 \theta}-H \frac{\mathrm{d}}{\mathrm{d} x} \ln u_{e}-(H+1) P \frac{\mathrm{d}}{\mathrm{d} x} e^{\left(x-x_{x}\right) / \delta}
$$

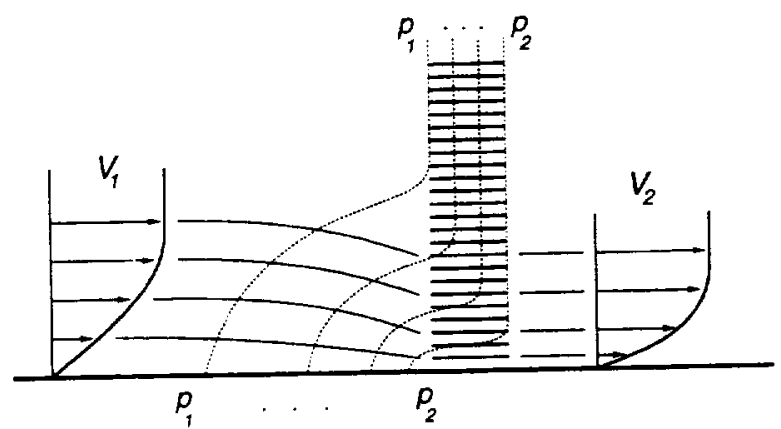

Fig. 4 Velocity profiles and pressure field associated with boundary layer passing through radiator.

$$
\begin{aligned}
& \frac{\mathrm{d}}{\mathrm{d} x} \ln H^{*}=\frac{2 c_{d}}{\theta H^{*}}-\frac{c_{f}}{2 \theta}+\left(H-1-\frac{2 H^{* *}}{H^{*}}\right) \frac{\mathrm{d}}{\mathrm{d} x} \ln u_{c} \\
& +\left(H-1-\frac{2 H^{* *}}{H^{*}}\right) \frac{P}{2} \frac{\mathrm{d}}{\mathrm{d} x} e^{\left(x-x_{r}\right) \delta}
\end{aligned}
$$

Here, $c_{f}$ and $c_{d}$ are the wall friction and dissipation coefficients, and $H, H^{*}$, and $H^{* *}$ are the conventional, kinetic energy, and density thickness shape parameters, respectively, as discussed by Drela and Giles. ${ }^{15}$ The effect of the new additional terms containing $P$ is dramatic. Even assuming the small value $P \simeq$ 1.5 , a roughly threefold reduction in the momentum and displacement thicknesses across the radiator is predicted for turbulent flow, consistent with the experimental observations mentioned by Mehta. ${ }^{14}$ This strong effect can be examined by neglecting the skin friction and any $u_{e}$ variations just upstream of the radiator, in which case Eq. (29) becomes

$$
\mathrm{d}(\ln \theta) \simeq-(H+1) P \mathrm{~d}\left[e^{\left(x-x_{r}\right) \delta}\right]
$$

Integrating this for the typical values $H=1.5, P \simeq 10$ shows that enormous decreases in the momentum thickness are possible:

$$
\frac{\theta_{1}}{\theta_{\text {upasream }}} \approx \exp [-(H+1) P] \simeq 10^{-11}
$$

In practice, the momentum thickness is virtually eliminated at the radiator, regardless of the particular choice of the length scale $\delta\left(=\delta^{*}+5 \theta\right.$ is assumed). In fact, the numerical implementation requires that $P$ be artificially limited to moderate values, e.g., $P<3$, to avoid numerical difficulties with a nearly vanishing boundary-layer thickness.

As a design consideration, this boundary-layer thinning behavior has a very beneficial effect in that the radiator has a strong tendency to suck out any separated fluid ahead of it, equalizing the flow rate all across the radiator face. This makes the overall configuration behavior relatively insensitive to the detailed design of the interior duct contours.

\section{Numerical Model Validation}

Figure 5 shows the computed streamline grid and $C_{p}$ contours for the integrated airfoil/heat-exchanger geometry used on the Perseus Unmanned Air Vehicle. The computation corresponds to one of a set of wind-tunnel tests aimed at measuring the performance of the overall configuration. ${ }^{16} \mathrm{~A}$ wire screen with a measured $P=6.7$ was employed to simulate the radiator on the tunnel model. The heat rejection of the actual Perseus radiator was not simulated, but this has little impact on the aerodynamics in this case. Figure 6 shows the computed surface $C_{p}$ distributions and compares the computed and measured drag forces. The computation separates the overall drag (momentum defect) into contributions from the viscous wake and from the inviscid radiator core flow wake:

$$
C_{D}=C_{\text {Drex }}+C_{D_{\text {mon }}}
$$



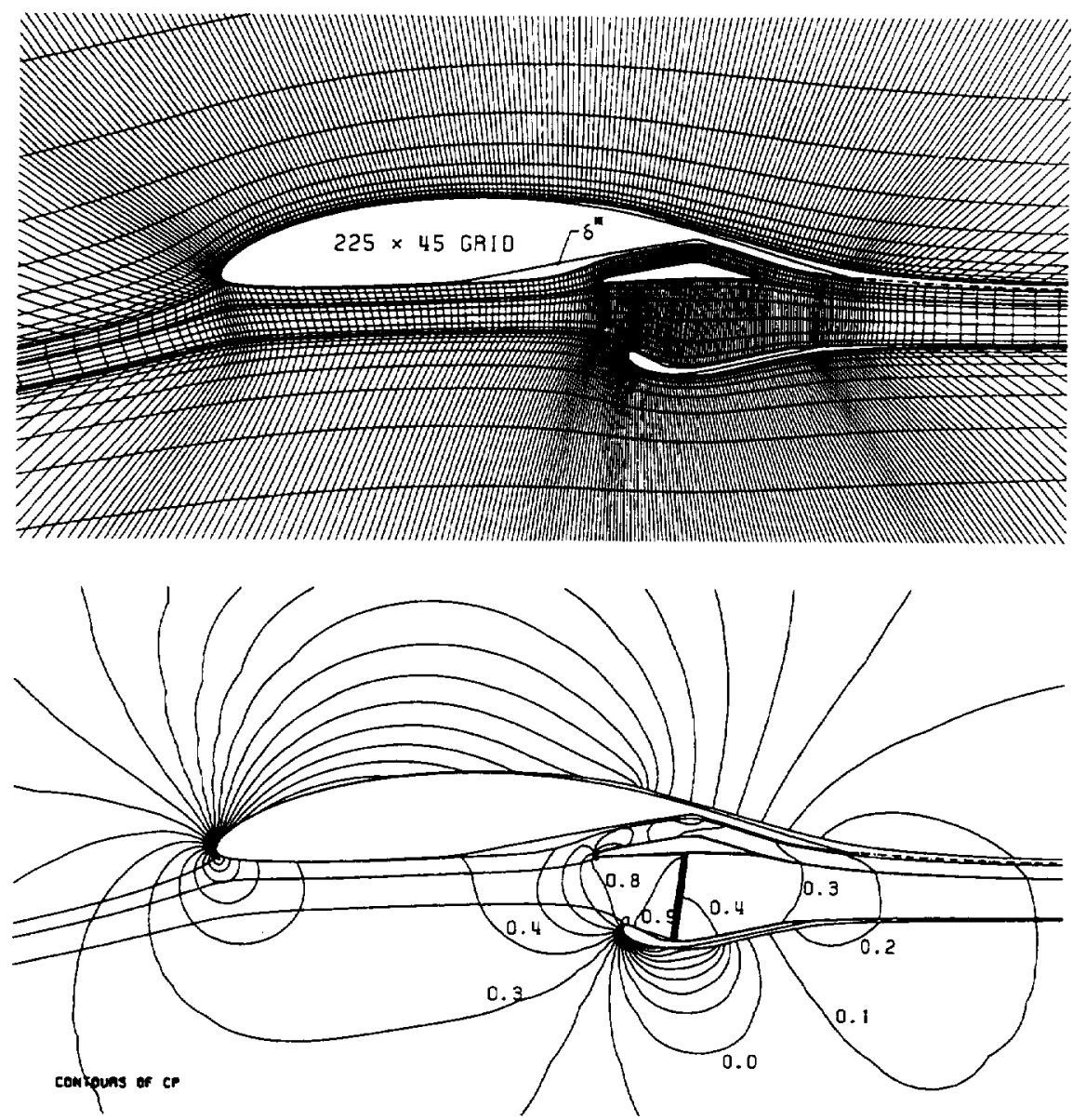

Fig. 5 Computed streamline grid and $C_{\text {, }}$ contours for Perseus airfoil/heat-exchanger test model configuration.

$$
\begin{gathered}
C_{D_{\text {vilc }}}=\frac{2}{c} \sum_{\text {elements }} \theta_{\text {visc }} \\
C_{D_{\text {core }}=} \frac{2}{\rho_{\infty} V_{\infty}^{2} c} \int_{\text {coreflow }}\left(V_{\infty}-V\right) \mathrm{d} m
\end{gathered}
$$

In the experiment, the total $C_{D}$ was measured by a wake rake spanning the entire viscous and core flow profile, and $C_{D_{\text {core }}}$ was deduced from the static and total pressure coefficients $C_{p_{2}}$ and $C_{p_{2}}$ measured immediately behind the radiator screen. With the assumptions that $C_{p 0_{1}}=1$ in front of the radiator, and that no further total pressure loss occurs in the core flow downstream of the radiator, Eq. (1) reduces to

$$
C_{D_{\text {cort }}}=2(h / c) \sqrt{C_{p 0_{2}}-C_{p_{2}}}\left(1-\sqrt{C_{p 0_{2}}}\right)
$$

where $h / c$ is the radiator height to reference chord ratio. Figure 6 shows that both the total $C_{D}$ and $C_{D_{\text {con }}}$ are accurately predicted. The rather low Reynolds number $R e=3 \times 10^{3}$ causes substantial transitional separation bubbles to appear on all surfaces. The single static pressure measurement behind the radiator screen matches the computation very well, indicating a correct prediction of the radiator air mass flow. Figure 7 compares computed polars for $R e=3 \times 10^{5}$ and $2.5 \times 10^{5}$ with measurements at three angles of attack. The agreement is very satisfactory, giving confidence to using the present numerical simulation method for investigating design issues in integrated airfoilheat-exchanger configurations.

\section{Integrated Airfoil/Heat-Exchanger Installations}

Although numerous types of radiator installations have been employed on piston-driven aircraft, on high-altitude aircraft the options have been more limited due to the sheer size of the radiators. The Boeing Condor ${ }^{4}$ and the Strato- $2 \mathrm{C}^{3}$ have their radiators contained entirely in large nacelles mounted on the wing. The Perseus UAV has its radiators inside a cowl system integrated with the aft portion of the wing airfoil, as shown earlier. This design was a retrofit into an existing wing structure and the minimal impact of the aft-mounted instaliation on the wing airfoil was a strong constraint. The Theseus UAV currently in development will employ an alternative integrated installation, with the cowl and radiator near the leading edge. Here, the presence of the radiator and cowl requires significant redesign of the local airfoil. The Perseus and Theseus aircraft are designed for ceilings in excess of $25 \mathrm{~km}$, and the isolated nacelles required to house their radiators would have been impractically large and incompatible with the payload and engine placement.

The aerodynamic advantage of an integrated airfoil/heat-exchanger design lies in the fact that the apparent freestream velocity seen by the cowl/radiator system can be reduced by the presence of the wing airfoil. If the cowl/radiator configuration shown in Fig. 1 is placed on the bottom of the wing airfoil, then the $V_{\infty}$ in Fig. 1 is reduced to something less than the true freestream velocity by the airfoil circulation. The cowl then experiences lower dynamic pressures, and hence, lower profile drag. Also, the amount of flow deceleration necessary to reach the required radiator velocity $V_{1}$ is smaller, allowing the cowl chord to be reduced, leading to further weight and drag reductions. On the other hand, integrated airfoil/heat-exchanger installations can give rise to unintended boundarylayer separation that must be controlled with careful design.

\section{A. Aft-Mounted Underwing Cowl}

Locating the heat exchanger at the airfoil aft bottom surface as with the Perseus configuration shown in Fig. 6 is structur- 


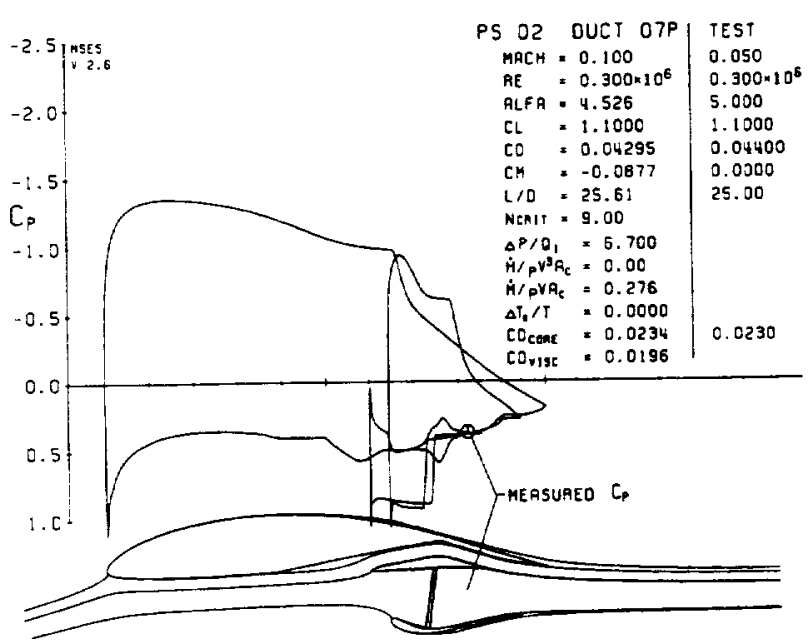

Fig. 6 Computed $C$, distributions and comparison of computed and experimental $C_{D}$ for Perseus airfoilheat-exchanger test model configuration.

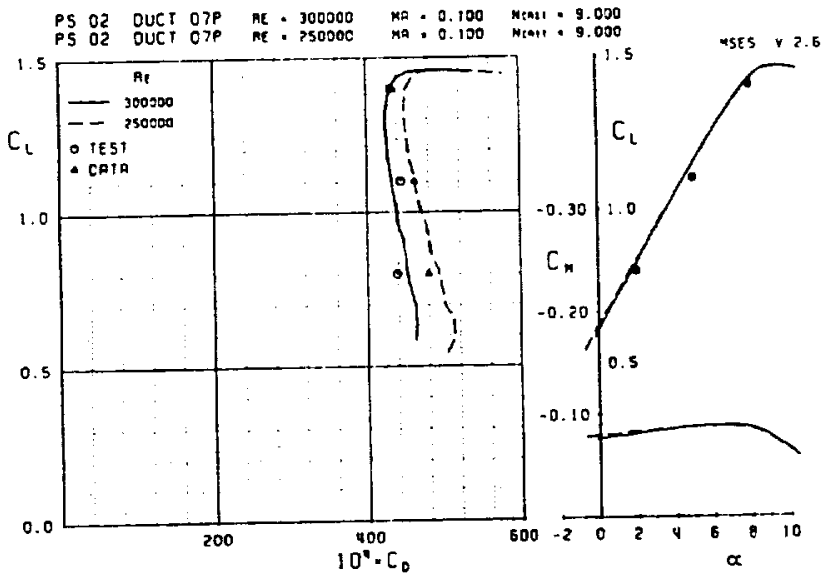

Fig. 7 Comparison of computed drag polars with measurements for test model of Perseus wing-airfoil/heat-exchanger configuration.

ally unintrusive and is attractive from potential-flow considerations. The local flow direction is essentially independent of the aircraft's angle of attack, and the local effective freestream velocity seen by the cowl can be as small as $0.8 V_{\infty}$ for aftloaded airfoils. The biggest source of difficulty is the wing bottom surface boundary layer, which cannot possibly negotiate the deceleration to the near-stagnation pressure at the radiator face. The suckdown effect examined earlier alleviates this to a significant extent, but its range extends only over a few boundary-layer thicknesses, and hence cannot suppress separation well upstream of the radiator. An effective solution is a splitter surface, which allows the wing boundary layer to bypass most of the cowl's pressure field.

An effective solution is a splitter surface, which allows the wing boundary layer to bypass most of the cowl's pressure field. Numerical investigations indicate that the length and positioning of the splitter is important. Figure 8 shows the integral heat exchanger installation of the Perseus aircraft with three splitter length versions, together with computed displacement bodies and several velocity profiles. The short splitter doesn't sufficiently protect the wing boundary layer from separation, whereas the long splitter has a thicker boundary layer, which itself separates. The intermediate splitter length strikes a reasonable balance between separation on the wing and splitter, and on this basis was selected for Perseus.

At the low Reynolds numbers being considered, the largest drawback of the aft-mounted heat exchanger installation ap- pears to be the difficulty of obtaining a radiator velocity ratio $V_{1} / V_{\text {o }}$ much below 0.2 without incurring significant separation either on the airfoil or the splitter. As a result, the overall drag level of the airfoil/heat-exchanger combination shown in Figs. 6 and 7 is three to four times that expected of the clean airfoil at the same operating conditions.

\section{B. Front-Mounted Underwing Cowl}

Most of the problems inherent in the aft-mounted radiator can be largely overcome with the front-mounted installation, which does not subject any thick boundary layer to the cowl radiator's pressure field and allows much smaller velocity ratios to be used. Figure 9 shows the airfoil/cowl/radiator configuration being developed for the large unmanned Theseus aircraft, which has a $27-\mathrm{km}$ design ceiling and is being targeted for atmospheric science missions. This configuration is a log ical extension of the leading-edge radiator intake commonly
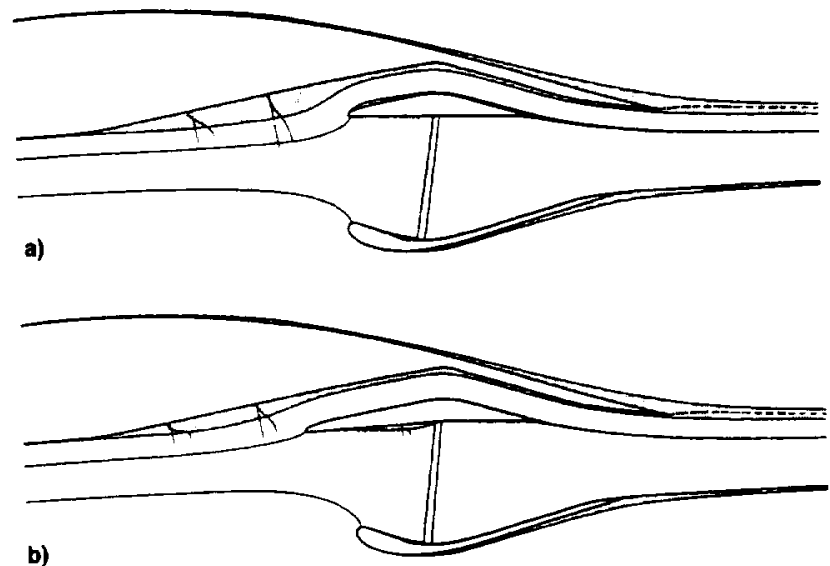

b)

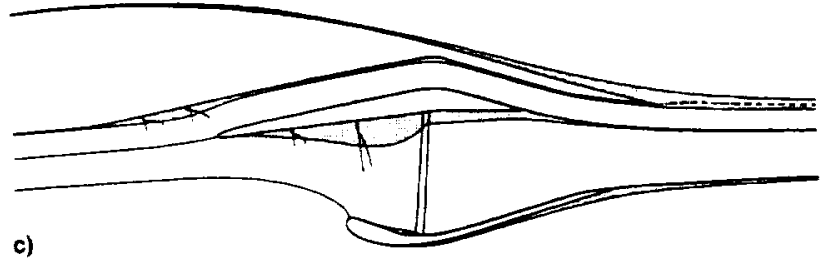

Fig. 8 Displacement bodies (shaded) and velocity profiles for Perseus airfoilheat-exchanger installations with various splitter surface lengths, at typical near-ceiling flight conditions: $\alpha=5$ deg, $\left.\left.R e_{c}=5 \times 10^{5}, M_{-}=0.4, h / \rho V^{3} A_{r}=0.5 . m / \rho V A_{r}=a\right) 0.242, b\right)$ 0.240 , and c) 0.223 .

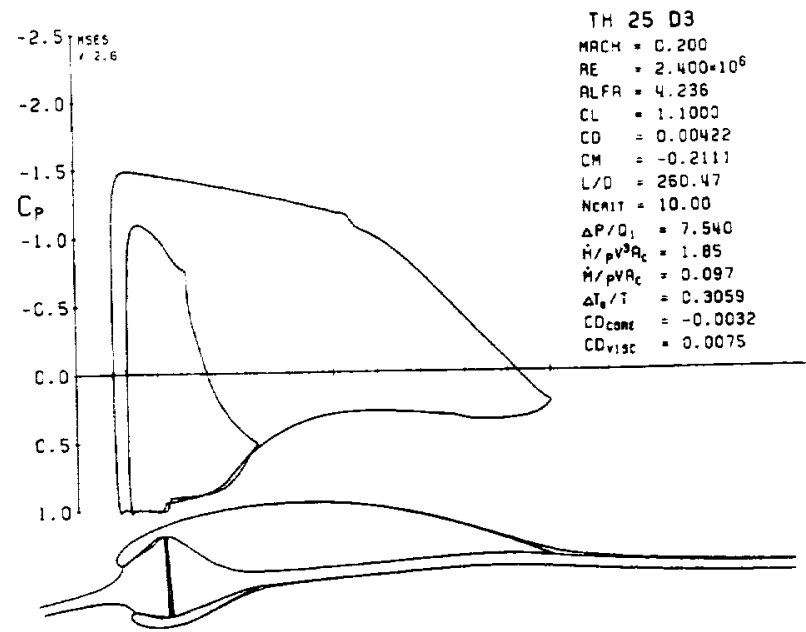

Fig. 9 Computed $C$, distributions for Theseus airfoil/heat-exchanger configuration. 


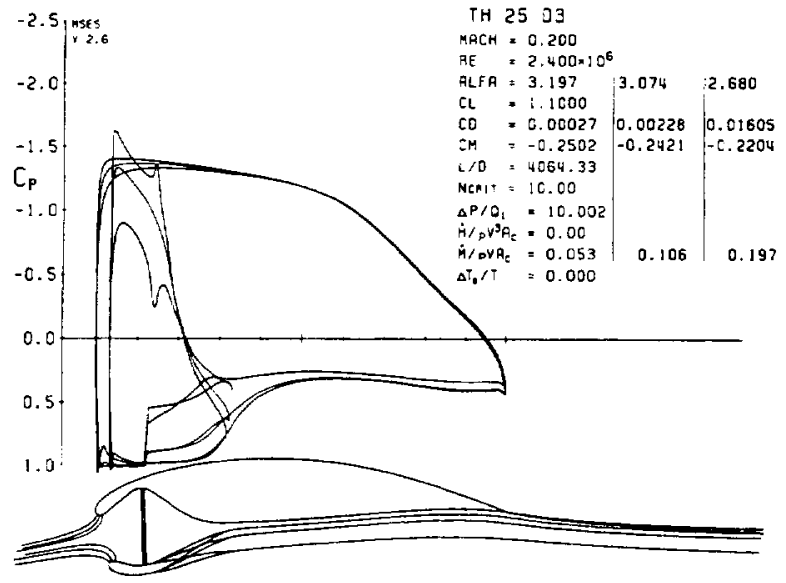

Fig. 10 Captured streamtube and surface $C_{\text {, }}$ variation for $V_{1} / V_{\text {. }}$ $=0.05,0.10$, and 0.20 adjusted via cowl flap position.

used on WW II aircraft, but with roughly four times larger radiator frontal area. Since most of the flow deceleration occurs ahead of the intake, there is little problem with flow separation, and velocity ratios $V_{1} / V_{\infty}$ less than 0.1 can be readily obtained. In the calculation in Fig. 9, which corresponds to operation near the tropopause, the radiator core drag is virtually nil even at the rather high pressure drop coefficient $P=$ 10 , most of the frictional drag being cancelled by the ramjet effect.

With the large variations in the velocity ratio $V_{1} / V_{\infty}$ required between the tropopause and ceiling (Fig. 3), the detailed design of the duct intake lip shapes is quite important. Figure 10 shows the behavior of the captured streamtube and the corresponding migration of the stagnation points as $V_{1} / V_{\infty} \simeq \dot{m} /$ $\rho_{\infty} V_{\infty} A_{\text {r }}$ is varied from 0.05 to 0.20 by adjusting the exit area via the cowl flap. Most of the surface pressure variation occurs on the cowl, which is favorable in that $C_{L_{\max }}$ of the overall section is only marginally affected. The presence of the cowl does reduce the $C_{\text {Lmax }_{\max }}$ relative to the clean airfoil, however, primarily due to the cowl's negative camber. A possible complication in the design of the cowl is the rather low cowlchord Reynolds numbers that can occur at ceiling. Fortunately, here the velocity ratio is typically set at its maximum and the pressure gradients on the cowl surface are most benign. A relatively open cowl flap will also incur the smallest $C_{L_{\text {mux }}}$ penalty on the airfoil, which is also favorable with regard to ceiling performance.

Wind-tunnel tests were performed on the Theseus frontmounted airfoil/radiator configuration to gain confidence that low velocity ratios could be attained at small chord Reynolds numbers. The cowl flap on the 10-in. chord model was hinged, allowing adjustment of the velocity ratios as would occur on an actual installation. A wire screen was again used to simulate the radiator. Figure 11 shows three drag polars corresponding to $0-, 5-$, and 10-deg flap settings, producing $V_{1} / V_{\infty}=0.11$, 0.14 , and 0.18 near the design condition of $C_{l}=1.1$. Each polar is at the same chord Reynolds number of $R e_{c}=4.5 \times$ $10^{5}$, roughly corresponding to a $27 \mathrm{~km}$ altitude. Also shown are the computed and measured (via stethoscope) upper-surface transition locations. These tests were of a preliminary nature, using a hand-built airfoil. The model airfoil differed somewhat from the design geometry, but the computations used the measured model coordinates for a direct comparison. A simple wire/strain-gauge balance used for the lift measurement and a total-pressure wake rake were used to measure the overall momentum defect. The experimental and computed $C_{D}$ curves in Fig. 11 actually represent the momentum thickness at $x / c=1.5$, where the wake rake was located, to permit a direct comparison. The far-downstream momentum defect, and hence the true $C_{D}$, is computed to be about $10 \%$ smaller.
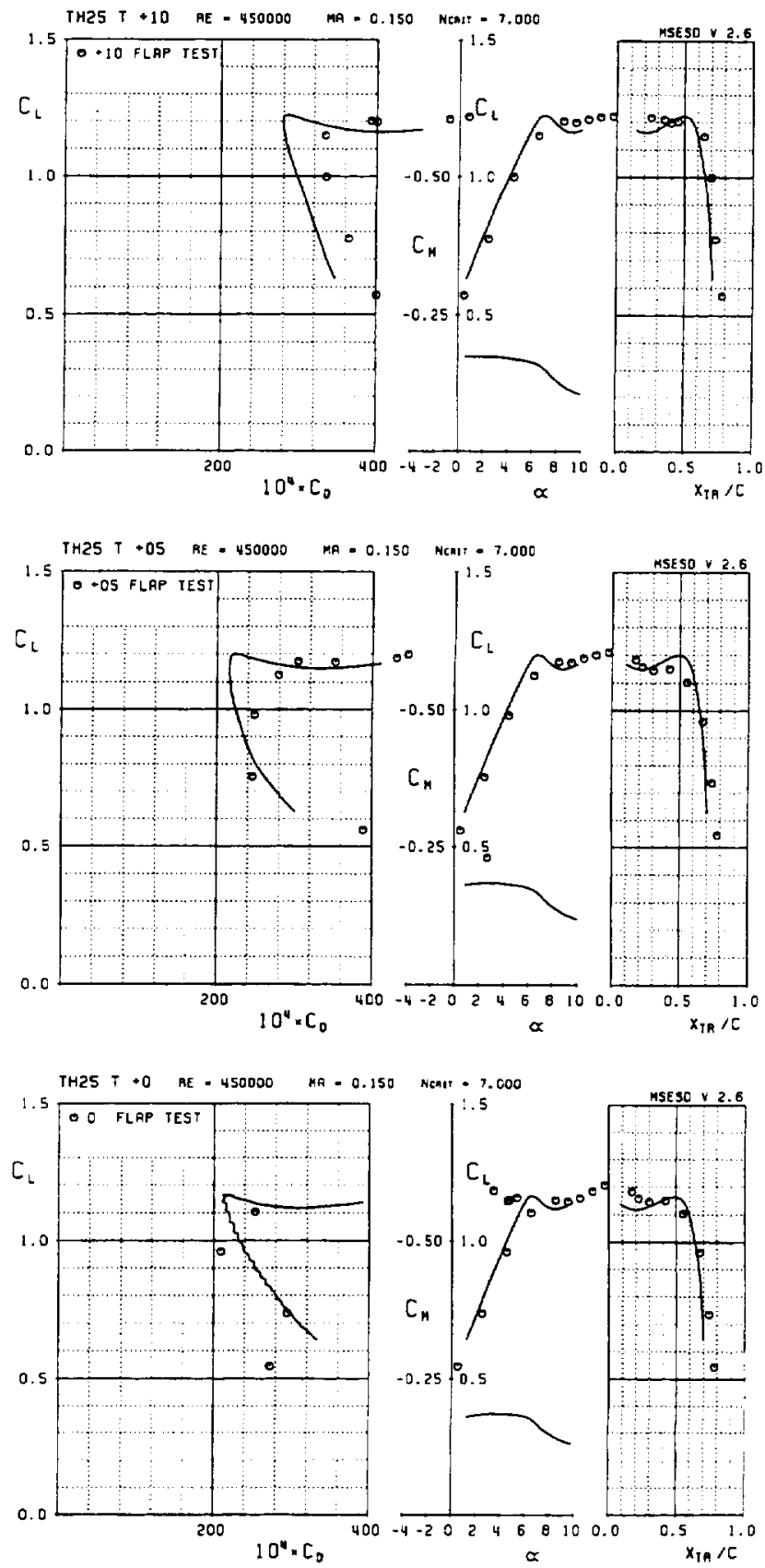

Fig. 11 Computed and experimental drag polars and upper-surface transition location of Theseus front-mounted radiator test configuration for 0-, 5-, and 10-deg cowl flap settings.

These modest experimental results clearly indicate that rather small velocity ratios are viable on low Reynolds number installations. The predicted sudden $C_{L}$ break at stall is not consistent with the data, but the rounding off of the drag polar near $C_{\text {Lmex }}$ is typical of hand-built models, which are prone to premature leading-edge $C_{p}$ spikes and consequent premature transition and separation at high angle of attack.

At the smallest velocity ratio $V_{1} / V_{\infty}=0.11$, at 0 -deg flap, some separation is incurred on the cowl, especially at the lower $\alpha$ values where a significant suction spike occurs on the cowl leading edge. Figure 12 shows the cowl separation, which matches the separation observed in the experiment using a stethoscope. The separation adds significantly to the viscous

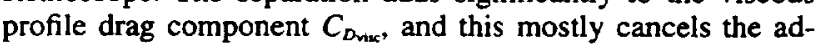
vantage of the smaller radiator drag component $C_{D_{\text {eor }}}$. However, this situation is not relevant for most applications, since 


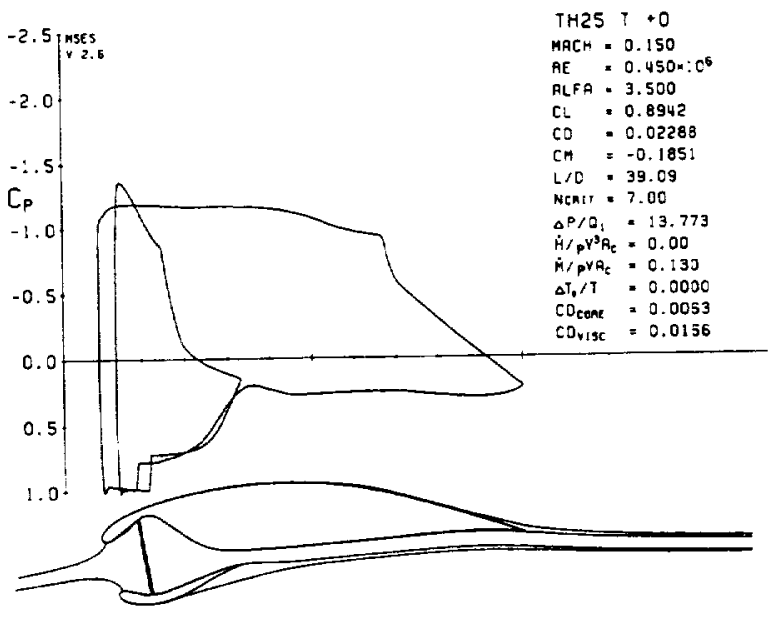

Fig. 12 Cowl separation induced by small veloctty ratio $V_{1} / V_{\text {. }}$ (closed cowl flap) and small Reynolds number.

such a small $V_{1} / V_{\infty}$ would only be used at lower altitudes and much higher Reynolds numbers where the cowl is much more resistant to separation, such as in the tropopause-altitude case shown in Fig. 9. These test results indicate the limit of using small velocity ratios at small Reynolds numbers.

\section{Conclusions}

This article has discussed the issues in the design of lowdrag heat exchanger installations for high-altitude aircraft. $\mathrm{Pa}$ rameters that characterize the effectiveness of such installations have been derived. The possibility of large drag penalties from poor installation designs has been shown to increase rapidly with altitude above the tropopause. The requirements of heat rejection regulation via variable geometry have also been examined.

A viscous/inviscid numerical simulation method for integrated airfoil/radiator flows has been demonstrated to be quite accurate at low chord Reynolds numbers and has proven useful in the development of candidate designs. The pros and cons of aft-mounted and front-mounted airfoil/heat-exchanger configurations have been examined with both computational results and some preliminary experimental data. The frontmounted configurations appear to be inherently superior. All integrated installations demand close attention to proper design.

\section{Acknowledgments}

This research was supported by the Massachusetts Institute of Technology T. Wilson Faculty Development Chair, and by
NASA Grant NAG2-4008 with Robert Geenen as Technical Monitor. Benjamin Russ of Aurora Flight Sciences provided valuable heat exchanger expertise, and Chris Anderson at MIT constructed the wind-tunnel model and test setups.

\section{References}

'Anderson, J. G., and Langford, J. S., "Unmanned Aircraft: An Essential Component in Global Change Research," Harvard Univ., Depts. of Chemistry and Earth and Planetary Sciences, Rept. V 1.0. Cambridge, MA, 1991.

${ }^{2}$ Langford, J. S., Drela, M., Driver, D., Hutchison, M., Kesseli, J., Preston, C., and Vos, D., "Theseus: A High-Altitude Aircraft for Atmospheric Science." Aurora Flight Sciences Corp., NSF SBIR Phase II Rept. Grant ISI-9103864, Manassas, VA, Nov. 1993.

${ }^{3}$ Tonskötter, H., "The STRATO-2C Propulsion System-A Low Cost Approach for a High-Altitude Long Endurance Aircraft." Recent Advances in Long Range and Long Endurance Operation of Aircraft. CP-547, AGARD, Nov. 1993, pp. 14.1-14.6.

"Henderson, B. W., "Boeing Condor Raises UAV Performance Levels," Aviation Week \& Space Technology, April 23, 1990, pp. 36 -38 .

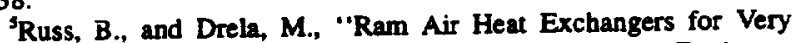
High-Altitude Subsonic Aircraft," Society of Automotive Engineers Paper 931145 , Sept. 1993.

'Harris, T. A., and Recant, I. G., "Investigation in the 7- by 10 Foot Wind Tunnel of Ducts for Cooling Radiators Within an Airplane Wing," NACA TR 743, 1942.

'Dannenberg, R. E., "A Design Study of Leading-Edge Inlets for Unswept Wings," NACA TR 3126, March 1954.

"Capon, R. S., "The Cowling of Cooling Systems," R\&M Rept. 1702, Aeronautical Research Council, HMSO, London, 1936.

'Hoerner, S. F., Fluid-Dynamic Drag, Hoerner Fluid Dynamies, Brick Town, NJ, 1965.

${ }^{10}$ Kays, W. M., and London, A. L., Compact Heat Exchangers, McGraw-Hiil, New York, 1984.

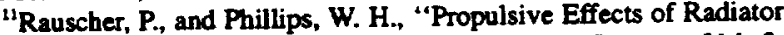
and Exhaust Ducting," Joumal of the Aeronautical Sciences, Vol. 8, No. 11, 1940, pp. $167-174$.

${ }^{12}$ McGeer, T., Langford, J. S., Anderson, J. G., and Drela, M., "Theseus: A High-Altitude Aircraft for Atmospheric Science," Aurora Flight Sciences Corp., Rept. AR 905, Manassas, VA, Nov. 1990.

${ }^{13}$ Drela, M., "Newton Solution of Coupled Viscous/Inviscid Multielement Airfoil Flows," AIAA Paper 90-1470, June 1990.

"Mehta, R. D.. "The Aerodynamic Design of Blower Tunnels," Progress in Aerospace Sciences, Vol. 18, 1977, pp. 59-120.

${ }^{15}$ Drela, M., and Giles, M. B., "Viscous-Inviscid Analysis of Transonic and Low Reynolds Number Airfoils," AIAA Journal, Vol. 25. No. 10, 1987, pp. 1347-1355.

16 Pouliot, C., and Glenn, C., "Aerodynamic Analysis of Heat Exchanger in the Perseus Aircraft," Dept. of Aeronautics and Astronautics, Experimental Projects II Course Lab Rept., Massachusetts Inst. of Technology, Cambridge, MA, Dec. 1992. 